\title{
Sustaining Canadian Medicare
}

He Underlying PRINCIPLE OF CANADian MediCARE - ACCESS to
essential care based on need - commands the enduring loyalty of the
Canadian public. How is it, then, that this principle remains insecure, and that attempts to extend it beyond physician and hospital services repeatedly falter?

Despite much noise to the contrary, the issue is not one of affordability. The claim that the Canadian model of publicly funded healthcare is, or will soon become, fiscally unsustainable has been resoundingly debunked (see, e.g., Evans 2005, 2006; Lewis 2007). If the United States can "sustain" a healthcare system that costs almost twice as much as Canada's and accounts for $50 \%$ more of its gross domestic product and a larger expenditure of public funds, maintaining (and expanding) Canadian medicare ought to be a walk in the park. ${ }^{1}$

Sincere adherents notwithstanding, the sustainability argument most often serves as a stalking horse for other objections to equity of access that reflect personal and class interests. A publicly funded and administered healthcare system forecloses opportunities for both sellers and purchasers of health-related goods and services. For sellers, the lost opportunities are even greater when healthcare is delivered by not-forprofit or public providers. And the more expansive the public system, the higher are the corporate and personal taxes required to support it.

A capitalist economy depends for its survival on economic growth. Capitalists are thus engaged in an unending search for potentially profitable investments. Not surprisingly, they take strong and persistent exception to limitations on the scope of their investment opportunities. Their objections to medicare's constraints are rarely expressed in terms of self-interest, but take the form of claims for the desirability of increased private (for-profit) sector involvement in healthcare financing and delivery - improved efficiency, expanded capacity, reduced wait times, lower taxes (for others), sustainability, greater "consumer choice" and so on. All this in the context of the "big idea" that what's good for the corporate sector is good for us all - the trickle-down theory, described by John Kenneth Galbraith as "the less than elegant metaphor that if one feeds the horse enough oats, some will pass through to the road for the sparrows" (Galbraith 1992). As Evans (2006) has pointed out, during the past 20 years in Canada, almost nothing has trickled down.

The claims that private financing and delivery of healthcare will achieve superior outcomes at reduced costs and lower wait times are grounded much more in ideology 
than in evidence. For example, a growing body of recent evidence points to the higher costs and poorer outcomes of for-profit healthcare (e.g., Himmelstein et al. 1999;

Devereaux et al. 2002a,b, 2004).

Permitting a parallel system of private insurance for services covered under provincial/territorial public plans, as contemplated by the majority in the Supreme Court of Canada's Chaoulli decision (2005), would create investment opportunities in health insurance and for-profit healthcare. On ideological grounds, such a development would likely be seen by the business community as a welcome breach in the dyke of legislative and regulatory restraint. However, at a practical level, recognizing that employers might be pressed to provide parallel private insurance for their employees - thereby incurring increased labour costs and impairing international competitiveness - corporate Canada might be divided on this issue.

The prospect of parallel private insurance appeals to the self-interest of those who can afford to pay for it, or who are likely to receive it as an employee benefit. They could expect to receive speedier access to needed physician and hospital services and, potentially, to "boutique" health services not currently available through provincial/territorial plans. However, given the Canadian public's continuing commitment to the idea of access to care based on need, many who could benefit personally may fail to rally behind parallel private insurance because of a commitment to social solidarity - particularly in light of the risk that large numbers of already scarce healthcare providers will be lured from the public to the private system, resulting in reduced access to needed care for those who are left behind.

The fundamental threat to Canadian medicare is not the challenge of "sustainability" but the interests of corporate Canada, which run counter to the principle of access to healthcare based on need and to the policies that support it. While the corporate claims may be shaky, they are the stuff and substance of media commentary and political discourse. Big business wields enormous economic and political power, both directly and through the media and its support of political candidates. Its use of that power has held medicare in check and threatens to roll it back.

Preserving and strengthening Canadian medicare will require shaking off the notion that what serves big business (usually referred to by mainstream politicians and the media as "the economy") necessarily serves the public interest. Only an unrelenting mobilization of public opinion behind a humane and communitarian vision of health and healthcare - a vision grounded in a commitment to equity - will allow medicare to survive and flourish. Civil society has its work cut out for it.

\section{Note}

1. In 2006, total health expenditures per capita measured in US dollars PPP (purchasing parity power) were $\$ 6,714$ in the United States and $\$ 3,678$ in Canada, 
and accounted for $15.3 \%$ and $10.0 \%$ of GDP, respectively. Public spending per capita on health was $\$ 3,075$ in the United States and \$2,589 in Canada. Source: IRDES (Institute for Research and Information in Health Economics. Retrieved October 16, 2008. <http://www.irdes.fr/EcoSante/Download/ OECDHealthData_FrequentlyRequestedData.xls $>$.

\section{REFERENCES}

Chaoulliv. Quebec (Attorney General), 2005 SCC 35.

Devereaux, P.J., P.T-L. Choi, C. Lachetti, B. Weaver, H.J. Schünemann, T. Haines, J.N. Lavis, B.J.B. Grant, D.R.S. Haslam, M. Bhandari, T. Sullivan, D.J. Cook, S.D. Walter, M. Meade, H. Khan, N. Bhatnagar and G.H. Guyatt. 2002a. "A Systematic Review and Meta-analysis of Studies Comparing Mortality Rates of Private for-Profit and Private Not-for-Profit Hospitals." Canadian Medical Association Journal 166: 1399-1406.

Devereaux, P.J., H.J. Schüneman, N. Rwvindran, M. Bhandari, A.X. Garg, P.T.-L. Choi, B.J.B. Grant, T. Haines, C. Lacchetti, B. Weaver, J.N. Lavis, D.J. Cook, D.R.S. Haslam, T. Sullivan, G.H. Guyatt. 2002b. "Comparison of Mortality between Private-For-Profit and Private Not-For-Profit Hemodialysis Centers." Journal of the American Medical Association 288(19): 2449-57.

Devereaux, P.J., D. Heels-Ansdell, C. Lachetti, H.J. Schünemann, N. Ravindran, M. Bhandari, A.X. Garg, P.T.-L. Choi, B.J.B. Grant, T. Haines, C. Lacchetti, B. Weaver, J.N. Lavis, D.J. Cook, D.R.S. Haslam, T. Sullivan, G.H. Guya. 2004."Payments for Care at Private For-Profit and Not-for-Profit Hospitals: A Systematic Review and Meta-analysis." Canadian Medical Association Journal 170(12): 1817-24.

Evans, R. 2005. "Baneful Legacy: Medicare and Mr. Trudeau." Healthcare Policy 1(1): $20-25$.

Evans, R. 2006. "From World War to Class War: The Rebound of the Rich." Healthcare Policy 2(1): $14-24$.

Galbraith, J.K. 1992. The Culture of Contentment. New York: Houghton Mifflin.

Himmelstein, D.U., S. Woolhandler, I. Hellander and S.M. Wolfe. 1999. "Quality of Care in Investor-Owned vs Not-for-Profit HMOs." Journal of the American Medical Association 282(2): $159-63$.

Lewis, S. 2007. "Can a Learning Disabled Nation Learn Healthcare Lessons from Abroad?" Healthcare Policy 3(2): 19-28.

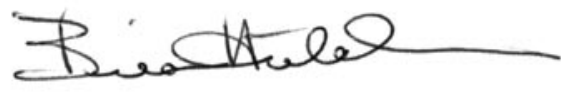

BRIAN HUTCHISON, MD, MSC, FCFP

Editor-in-chief 


\section{Préserver l'assurance maladie au Canada}

$\mathrm{L}$

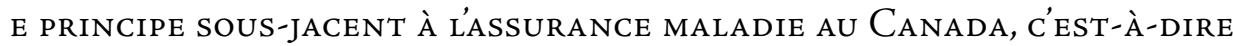
l'accès aux soins essentiels en fonction des besoins, implique une loyauté ferme de la part des Canadiens. Comment se fait-il, donc, que ce principe demeure précaire et que les tentatives pour l'appliquer au-delà des services médicaux ou hospitaliers échouent continuellement?

Malgré le battage qui nous entoure, il ne sagit pas en fait d'une question de moyens financiers. Lidée selon laquelle le modèle canadien de services de santé subventionnés par l'État est, ou deviendra bientôt, financièrement non durable a été vivement réfutée (voir, par exemple, Evans 2005, 2006; Lewis 2007). Si aux États-Unis on parvient à « soutenir » un système de santé qui coûte près du double de celui du Canada, un système qui représente plus de $50 \%$ du produit intérieur brut et qui implique de plus grandes dépenses de fonds publics, maintenir (et accroître) l'assurance maladie au Canada devrait être un jeu d'enfant ${ }^{1}$.

Mises à part les convictions sincères, la durabilité est un argument employé plus souvent comme prétexte pour d'autres objections à l'égalité d'accès, lesquelles reflètent les intérêts personnels et des classes. Un système de services subventionné et administré par l'État prive de possibilités les vendeurs et les acheteurs de biens et services liés à la santé. Pour les vendeurs, la perte de possibilités est encore plus grande si les services sont fournis par des organismes publics ou sans but lucratif. Et plus le système public est généralisé, plus élevés sont les impôts personnels ou d'entreprises nécessaires pour le maintenir.

Pour survivre, une économie capitaliste est tributaire de la croissance économique. Les financiers sont donc continuellement à la recherche d'investissements potentiellement avantageux. Il n'est donc pas surprenant de voir qu'ils s'opposent fermement aux limitations qui peuvent toucher les occasions d'investissement. Leurs objections face aux contraintes en matière d'assurance maladie s'expriment rarement en termes d'intérêts personnels, mais se présentent sous forme de revendications visant une plus grande participation du secteur privé (à but lucratif) dans le financement et la prestation des services de santé - efficacité accrue, plus grande capacité, réduction des temps d'attente, baisse d'impôts (pour les autres), durabilité, meilleur choix pour les consommateurs, etc. Le tout présenté dans le contexte affirmant que ce qui est bon pour l'entreprise est bon pour tous, soit l'« effet de diffusion » décrit par John Kenneth Galbraith comme « la métaphore inélégante voulant que si l'on fournit suffisamment d'avoine à un cheval, quelques grains tomberont sur la route pour les oiseaux » (Galbraith 1992). Comme l'a indiqué Evans (2006), on n'observe pas une telle diffusion au cours des 20 dernières années.

L'idée acceptant que la privatisation du financement et de la prestation des services 
de santé permettra de meilleurs résultats à moindre coûts tout en réduisant les temps d'attente śappuie sur une idéologie plutôt que sur des données probantes. Par exemple, de plus en plus de données récentes démontrent que les organismes de santé à but lucratif obtiennent de plus faibles résultats à un coût plus élevé (voir, par exemple, Himmelstein et al. 1999; Devereaux et al. 2002a,b, 2004).

Permettre un système de santé privé parallèle pour des services subventionnés par le gouvernement, tel qu'envisagé dans la décision majoritaire de la Cour suprême pour la cause Chaoulli (2005), créerait des occasions d'investissement pour l'assurance maladie privée et pour les organismes de santé à but lucratif. Du point de vue idéologique, une telle ouverture serait probablement accueillie par le milieu des affaires comme une percée dans les restrictions et les règlements juridiques. Toutefois, au niveau pratique, le milieu des entreprises canadiennes pourrait être divisé sur cette question puisque les employeurs se sentiraient sans doute obligés d'offrir aux employés une assurance privée parallèle, augmentant ainsi les coûts liés à la main-d’œuvre et réduisant la capacité concurrentielle à l'échelle internationale.

La perspective d'une assurance privée parallèle est intéressante pour ceux qui ont les moyens de payer, ou qui peuvent en bénéficier dans le cadre de leurs avantages sociaux. Ils peuvent sattendre à un accès plus rapide aux médecins et aux services hospitaliers et, possiblement, à un accès à des services de santé qui ne font pas partie actuellement des régimes d'assurance provinciaux ou territoriaux. Toutefois, étant donné l'engagement profond des Canadiens envers un accès aux soins en fonction des besoins, plusieurs parmi ceux qui en bénéficieraient personnellement pourraient hésiter à approuver l'assurance privée parallèle pour des raisons de solidarité sociale - particulièrement face au risque que de nombreux fournisseurs de santé, déjà en situation de pénurie, soient attirés vers le système privé, réduisant ainsi l'accès aux soins pour les autres.

La principale menace à l'assurance maladie au Canada nest pas la question de la « durabilité », mais bien celle des intérêts des entreprises canadiennes, qui vont à l'encontre des politiques et du principe de l'accès aux soins en fonction des besoins. Même si les allégations des entreprises demeurent boiteuses, ce sont néanmoins elles qui constituent le matériau des messages médiatiques et du discours politique. Les grandes entreprises exercent avec autorité un énorme pouvoir économique et politique, que ce soit directement ou par l'entremise des médias, ou encore par l'appui de candidats politiques. L'exercice de ce pouvoir freine l'assurance maladie et risque de la faire régresser.

Pour préserver et renforcer l'assurance maladie au Canada, il faut s'attaquer à l'idée voulant que ce qui est bon pour les grandes entreprises (que les principaux politiciens et les médias appellent « l'économie ») est nécessairement bon pour l'intérêt public. Seule une mobilisation déterminée de l'opinion publique, accompagnée d'une vision humaniste et communautaire des services de santé - une vision ancrée dans un engagement envers l'égalité -, permettra la survie et l'essor de l'assurance maladie. La société civile a bel et bien du pain sur la planche. 


\section{Note}

1. En 2006, les dépenses totales de santé, par tête, calculées selon la parité des pouvoirs d'achat (PPA) en dollars US, étaient de l'ordre de 6714 \$ aux États-Unis et de 3678 \$ au Canada; et elles comptaient, respectivement, pour 15,3\% et 10,0\% du PIB. Les dépenses publiques de santé, par tête, étaient de 3075 \$ aux ÉtatsUnis et de 2589 \$ au Canada. Source: IRDES (Institut de recherche et documentation en économie de la santé). Consulté le 16 octobre 2008. <http://www.irdes. fr/EcoSante/Download/OECDHealthData_FrequentlyRequestedData.xls $>$.

\section{RÉFÉRENCES}

Chaoulliv. Quebec (Attorney General), 2005 SCC 35.

Devereaux, P.J., P.T-L. Choi, C. Lachetti, B. Weaver, H.J. Schünemann, T. Haines, J.N. Lavis, B.J.B. Grant, D.R.S. Haslam, M. Bhandari, T. Sullivan, D.J. Cook, S.D. Walter, M. Meade, H. Khan, N. Bhatnagar and G.H. Guyatt. 2002a. "A Systematic Review and Meta-analysis of Studies Comparing Mortality Rates of Private for-Profit and Private Not-for-Profit Hospitals." Canadian Medical Association Journal 166: 1399-1406.

Devereaux, P.J., H.J. Schüneman, N. Rwvindran, M. Bhandari, A.X. Garg, P.T.-L. Choi, B.J.B. Grant, T. Haines, C. Lacchetti, B. Weaver, J.N. Lavis, D.J. Cook, D.R.S. Haslam, T. Sullivan, G.H. Guyatt. 2002b. "Comparison of Mortality between Private-For-Profit and Private Not-For-Profit Hemodialysis Centers." Journal of the American Medical Association 288(19): 2449-57.

Devereaux, P.J., D. Heels-Ansdell, C. Lachetti, H.J. Schünemann, N. Ravindran, M. Bhandari, A.X. Garg, P.T.-L. Choi, B.J.B. Grant, T. Haines, C. Lacchetti, B. Weaver, J.N. Lavis, D.J. Cook, D.R.S. Haslam, T. Sullivan, G.H. Guya. 2004."Payments for Care at Private For-Profit and Not-for-Profit Hospitals: A Systematic Review and Meta-analysis." Canadian Medical Association Journal 170(12): 1817-24.

Evans, R. 2005."Baneful Legacy: Medicare and Mr. Trudeau." Healthcare Policy 1(1): 20-25.

Evans, R. 2006. "From World War to Class War: The Rebound of the Rich." Healthcare Policy $2(1): 14-24$.

Galbraith, J.K. 1992. The Culture of Contentment. New York: Houghton Mifflin.

Himmelstein, D.U., S. Woolhandler, I. Hellander and S.M. Wolfe. 1999. "Quality of Care in Investor-Owned vs Not-for-Profit HMOs." Journal of the American Medical Association 282(2): 159-63.

Lewis, S. 2007."Can a Learning Disabled Nation Learn Healthcare Lessons from Abroad?" Healthcare Policy 3(2): 19-28.

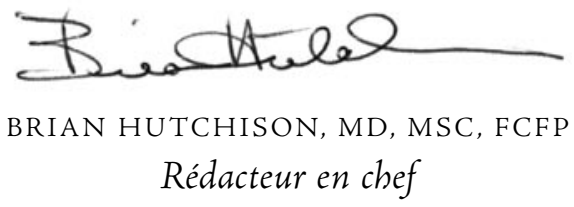

